Future internet foundations: Socio-economic issues - introduction

Stiller, Burkhard

DOI: https://doi.org/10.1007/978-3-642-20898-0

Posted at the Zurich Open Repository and Archive, University of Zurich

ZORA URL: https://doi.org/10.5167/uzh-55693

Book Section

Published Version

Originally published at:

Stiller, Burkhard (2011). Future internet foundations: Socio-economic issues - introduction. In: Domingue, John. The Future Internet - Future Internet Assembly 2011: Achievements and Technological Promises. Heidelberg: Springer, 117-119.

DOI: https://doi.org/10.1007/978-3-642-20898-0 


\section{Part II:}

Future Internet Foundations: Socio-economic Issues 


\section{Introduction}

Information and Communication Technologies (ICT) provide in recent years solutions to the sustainability challenge by, e.g., measuring impacts and benefits of economic activity via integrated environmental monitoring and modeling, by managing consequences, and by enabling novel low-impact economic activities, such as virtual industries or digital assets. In turn, ICT enables novel systems - in terms of technologies and applications - encouraging and generating socio-economic values. Additionally, these models address in many cases free-market forces, which may be likely ruled by governmental and regulatory acts. Thus, the inter-dependencies between global markets have never been greater and the global connectivity principle, underpinning the technology of the Internet, is particularly responsible for this accelerating trend.

Particularly, controlling and monetizing the evolution of the Internet and its vast application range is seen as a critical goal for most economic regions. Therefore, socio-economic aspects determine a highly important set of influencing factors, which are required to be understood for an in-depth and in-detail investigation of the economic viability and the social acceptability of modern technology and applications. While pure economic research as well as pure social research has been undertaken for decades, the combination of the two and its application to the new Internet - the one, which is rooted in the commercialization of the native research Internet of the early 90 's - becomes an important element in investigating, estimating, and understanding the risks, challenges, and usability aspects of this network of networks.

As collected by the FISE (Future Internet Socio-economics) working group within the FIA on its wiki, the following general aspects of socio-economics, particularly in networking, are considered to be important: (1) The study of the relationship between any sort of economic activity (here networking in the areas of Internet-based and telecommunications-based communications for a variety of lower-level network/telecommunication as well as application-based services) and the social life of user (here, mainly addressing private customers of such services and providers offering such services); (2) Markets of Internet Service Providers (ISP) and Telecommunication Providers; (3) ISPs peering agreements and/or transit contracts; (4) Customer usage behavior and selections of content; (5) The investigation of emerging technologies and disruptive technologies, which effect the user/customer-to-provider relation; (6) The investigation of (European) regulation for e-services markets and security regulations; (7) The investigation of the physical environment of e-services in terms of availability, world-wide vs. highly focused (cities), and dependability for commercial services; and (8) The determination (if possible) of the growth of the Gross Domestic Product (GDP), providers' revenue maximization, and customers' benefits. While this collection cannot be considered complete, it clearly outlines that a combination of social and economic viewpoints on pure Internet-based networking is essential.

Thus, the full understanding and modeling of these socio-economic impacts on Internet communications particularly and the Internet architecture generally challenges networking research and development today. Economic effects of technical mechanisms in a given setup and topology needs to be investigated and benefits obtained by optimizing or even changing existing protocols may lead to more cost- 
effective approaches. Furthermore, the users' perspectives need to be taken into close consideration, since detailed and specific security demands, electronic identities, or Quality-of-Experience (QoE) will outline societal requirements to be met by technological support means, while being at the same time in contrast to simplicity and easeof-operations of a variety of Internet-based services.

In this cmerging area of research the specific view on the networking and transmission domain of the Internet had been taken as one starting point of socio-economic rescarch for this FIA book. Thus, the content of these chapters on socio-economics of the Future Internet contains three views, where the decision of inclusion was based on two rounds of abstract reviews and on subsequent reviews of complete chapter proposals. The submission of in total six chapter proposals, addressing the socioeconomics domain, has shown that the interest of such cross-disciplinary work and its relevance increases slowly. While the first socio-economic chapter addresses aspects (1), (2), (4), and (8) as above, the second one works on (5) and (8). Finally, the last chapter tackles aspects (2), (3), and (8).

The first chapter by I. Papafili et al. is entitled "Assessment of Economic Management of Overlay Traffic - Methodology and Results". Due to the fact that overlay applications as of today still generate large volumes of data, Internet Service Providers (ISP) need to address the problem of expensive interconnection-charges. Thus, a reduction of inter-AS traffic (Autonomous Systems), which crosses domain boundaries of competitors, was tackled by an incentive-based approach, since traditional traffic management approaches do not deal with overlay traffic effectively. To ensure a mutually beneficial situation for all stakeholders in a Future Internet scenario, the "TripleWin" investigations determine the key goal of Economic Traffic Management (ETM) mechanisms developed. Thus, this chapter outlines the methodology developed, applied, and evaluated under a variety of constraints, which results in a detailed discussion of various ETM mechanisms.

The second chapter by E. Eardly et al. was submitted with the title "Deployment and Adoption of Future Internet Protocols". Based on the assumption that many welldesigned protocols designed for the Future Internet will fail - as it happened for the traditional Internet -, however, badly-designed ones are successfut. Thus, the problem of protocols' deployability is addressed. In order to do so, a framework had been developed, which includes the investigation possibilities for deployment effects, adoption characteristics, and their respective mechanisms. In a case-based study, the Multipath TCP (Transmission Control Protocol) and the Congestion Exposure approach are evaluated applying the framework developed. In turn, this chapter concludes that a careful consideration of certain parameters can increase the likelihood that a newly developed protocol, as it happens currently for the Future Internet, can get adopted.

Finally, the third chapter by C. Kalgoris et al. is on "An Approach to Investigating Socio-economic Tussles Arising from Building the Future Internet". Based on the assumption that the Internet has evolved into a world-wide social and economic platform with a variety of stakeholders involved, the key motivations of each of them and their behavior has changed over the recent past dramatically. In turn, conflicts have emerged, which are determined by opposing and contradicting interests. While this general problem had been characterized as "tussle" in the literature, it was decided to investigate, classify, and develop an analysis framework for such tussles in the socioeconomic domain of Internet stakeholders. In turn, the chapter outlines a ncw methodology, with which tussles are analyzed. Although a survey reveals that many tussles are known, neither of them are modeled in full nor even solved. Therefore, existing Future Networks projects in the FP7 program are identified for inputting existing tussles in order to provide for a structured analysis of social and economic aspects in a coherent and integrated manner on real-world examples.

Burkhard Stiller 